\title{
LA CONFIGURACION DE LA IDENTIDAD DE LOS SECTORES POPULARES EN LA ERA DE LA GLOBALIZACIÓN. APUNTES PARA PENSAR LAS PRÁCTICAS ESCOLARES
}

\author{
Natalia Jorgelina Forlini (UNR-CONICET)*
}

\begin{abstract}
Resumen
El presente escrito tiene como objetivo plantear la problemática de la configuración de la identidad de los sectores populares en el contexto actual y su vinculación con la escuela. Tomamos el aporte de las diferentes Ciencias Sociales y Humanas, pretendiendo conseguir desde ese lugar, el enriquecimiento de las prácticas educativas. El actual sistema de producción globalizado nos enfrenta a interpelaciones que se presentan, en apariencias, como nuevas cuestiones, nuevos enigmas a descifrar en las escuelas.

Proponemos pensar a la identidad, producida en el discurso del conjunto de los sectores populares, como un elemento clave para las instituciones educativas y las prácticas de enseñanza y aprendizaje; que busquen amplificar su mirada hacia una educación que gire en torno a estados emancipatorios del sujeto y su grupo de pertenencia.

Se parte de un posicionamiento social de la identidad para trazar discusiones conceptuales, que contribuyan con los debates involucrados en la problemática de la reproducción de cánones culturales en la escuela. Junto a ello, elaborar reflexiones e interrogantes acerca de las modificaciones que fueron transformando la escuela de la modernidad en la escuela actual, de la educación globalizada produciendo diferentes miradas escolares entorno a los sectores populares, su legado y su identidad.
\end{abstract}

\section{Palabras clave}

Identidad - Prácticas escolares - Sectores populares - Globalización - Reproducción de cánones culturales.

Licenciada y Profesora en Ciencias de la Educación. Facultad de Humanidades y Artes. Universidad Nacional de Rosario. Doctoranda en Ciencias de la Educación de la Facultad de Humanidades y Ciencias de la Educación de la Universidad Nacional de La Plata. Becaria Doctoral del CONICET (Tipo I) 


\begin{abstract}
This letter is intended to raise the issue of shaping the identity of the popular sectors in the current context and its relationship with the school. We take the contribution of different social and human sciences, aiming to get from there, the enrichment of educational practices. The current global production system we are faced with challenges that are presented in appearances, as new issues, new puzzles to decipher in schools.

We propose to think of identity produced in the speech of all the popular sectors as a key to educational institutions and teaching and learning practices that seek to amplify their gaze towards education that revolve around the subject and states emancipatory the group of belonging.

It is part of a social position to trace the identity of conceptual discussions that contribute to the discussions involving the issue of cultural reproduction in school fees. Along with, it to develop thoughts and questions about the changes that were transforming the school of modernity in the current school, global education schools producing different views around the popular sectors, his legacy and identity.
\end{abstract}

\title{
Key words
}

Identity - School practices - Popular areas - Globalization - Playing cultural canons.

\section{Introducción}

Historicidad, unidad, continuidad, hacen a ese proceso social y subjetivo denominado identidad. Ella es el soporte interno del sujeto y de las organizaciones. Desde su identidad que implica movimiento, semejanza y diferencia, el sujeto se sitúa en el presente y anticipa un futuro, sostenido y sosteniendo un proyecto que da sentido a su vida personal y a su ser social. Este proyecto será entonces su referente, su sostén. Desde el proyecto adquiere una dimensión de lo posible, una apertura y una capacidad anticipatoria con posibilidad de planificar la esperanza (Quiroga, 2005, p.29).

Es ampliamente conocido que el prestigio social de la ciencia moderna se funda en la garantía de verdad y utilidad del conocimiento. Desde la parcelación en la producción de dicho conocimiento, la comunidad científica ha buscado criterios de eficiencia, valores de uso y cambio, para dar garantía de veracidad y utilidad al conocimiento. De esta manera ha podido mantenerse en la posición detentadora del poder de la verdad científica, que le permite decidir cuáles son los conocimientos habilitados para ser considerados científicos y cuales no (Heler, 2004).

Tal planteo conlleva, precisamente, la idea de una división jerárquica y descendente al interior de cada ciencia; la misma se conforma con un nivel más 
alto de abstracción, donde se ubica a la denominada ciencia pura, asignándole el desarrollo del análisis, la teorización y la sistematización del conocimiento científico. En un segundo nivel, la ciencia aplicada, incluirá la exploración de soluciones prácticas; y por último, la tecnología abordará la operación, intervención y experimentación sobre dicho conocimiento en estudio (Ibídem).

No obstante, dentro del anteriormente mencionado panorama actual de las ciencias, se señala, además, que se están desplegando diversos movimientos entre las Disciplinas Sociales y Humanas, uno de ellos es la problemática de su demarcación; donde los límites entre una y otra disciplina comienzan a confundirse. Como si empezaran a compartir parte del conocimiento científico -el objeto de estudio, los aspectos metodológicos, los avances científicos- pasando a conformar "el desarrollo de espacios comunes,"de allí como derivación a la ampliación del campo disciplinario a la vez que la superposición con otros campos" (Bixio y Heredia, 2000, p. 87).

El campo científico de las Ciencias de la Educación -englobadas dentro de las Ciencias Sociales y Humanas- no escapa a las problemáticas que estamos planteando, es decir, que su desarrollo tropieza también con los condicionamientos de los movimientos históricos propios del desenvolvimiento del conocimiento científico que se produce en las diferentes disciplinas. Al no ser una estructura estática, o un sistema de "lugares vacíos," se podrían pensar como un conocimiento complejo y heterogéneo, un conjunto de saberes que epistemológicamente podrían tener características diferentes, que se plantean problemas disímiles, que tienen objetos de estudio diferentes, y metodologías diversas para el abordaje del conocimiento. Asimismo, entendemos que las Ciencias de la Educación, conformarían un conjunto de disciplinas que se construyen en tensión dialéctica permanente entre posicionamientos más relacionados con la interdisciplina, y quienes perciben relaciones entre las disciplinas que lo conforman atravesadas por un eje en común -la problemática educativa- para la construcción del objeto y su teorización, la transdisciplina y/o la multidisciplina.

En la tirantez de la discusión por la validación del conocimiento y los cuestionamientos a dichos postulados se desarrolla el presente trabajo. En el abordaje de este buscaremos que los límites entre las disciplinas se muestren maleables, para que el enfoque propuesto pueda tender no sólo al mejoramiento de la profundización del análisis, sino que también se corresponda con un tratamiento integral de la problemática que detallamos a continuación.

El presente trabajo es producto de los avances realizados en mi Tesis Doctoral titulada: Escuela, migrantes internos y comunidad barrial en Rosario. Cultura y socialización en la segunda mitad del siglo XX. Este proyecto de investigación tiene por objeto explorar en un barrio de la ciudad de Rosario, la incidencia de la escuela primaria en la identidad y en la herencia cultural de los pobladores migrantes internos, a través de los cánones culturales. Las 
entrevistas utilizadas pertenecen al material de campo del proyecto de investigación mencionado. Quedan en el anonimato tanto el nombre del barrio y de la escuela como el de los entrevistados.

Desde allí nos preguntamos, ¿Cómo pensar la constitución de una identidad cuando las organizaciones, fuentes del proceso de producción y reproducción del legado cultural y del colectivo social, se encuentran imbuidas con una maraña de nuevos conflictos que trastocan hasta su médula? En otras palabras, ¿cómo se configura la identidad dentro del actual sistema de producción global en donde hay un predominio de la individualización y de la destitución de las organizaciones de la modernidad, en su rol de conformadores de identidad social, cambiando definitivamente, o radicalmente el rol que les fue asignado en la modernidad: de coerción, vigilancia y adaptación del sujeto a cambio de un lugar de filiación en el grupo de pertenencia?

A partir de este escrito, pretendemos problematizar el desarrollo del concepto de identidad, no en un nivel abstracto sino encuadrado en las coordenadas del tiempo y del espacio, anclado en las manifestaciones concretas de los sectores populares de la ciudad de Rosario inmersos en la era de la globalización. Si a ello le sumamos que nuestro interés reside en inferir en el aporte, de estos dinamismos de la configuración de la identidad, al interior de las prácticas escolares; nos encontramos con una problemática más que frecuente aunque poco discutida; que no es lo mismo decir que no haya bibliografía al respecto.

En relación a nuestro tema de interés planteado up supra reunimos una serie de conceptos que nos permiten obtener un marco teórico referencial, para examinar desde allí, las prácticas escolares. Este será explicitado en el progreso del trabajo en función de las necesidades y profundizaciones de nuestro análisis, es decir, contrariamente a que la teoría sea el eje vector de la producción del conocimiento esta se halla desacralizada y puesta en acción como enriquecimiento de la reflexión sobre la práctica. Dicho postulado, por consiguiente, no implica la supremacía de la práctica sino que la tensión entre ambas, entendemos, sería la productora del conocimiento.

Algunos cuestionamientos vectores que inquietan a pensar/escribir en torno a estas problemáticas se relacionan con: ¿Por qué pensar en la identidad y no en la subjetividad? ¿Cuáles son sus diferencias? ¿Qué puede aportar esta discusión al trabajo en organizaciones educativas, tanto formales como no formales? Se trata de tomar el aporte de las diferentes Ciencias Sociales y Humanas para conformar una mirada educativa amplia que contribuya con el desanudamiento y el análisis de la discusión trazada, pretendiendo conseguir desde ese lugar, el enriquecimiento de las prácticas educativas con sectores populares. 


\section{Configuración del espacio histórico-social: De la sociedad de socorros mutuos a la sociedad anónima}

Una gran variedad de autores que no necesariamente parten de la misma línea teórica (Bauman, 1999, 2001; Beck, 1998; Dufour, 2007; Requejo, 2004; Racedo, 2004, etc.), ponen en cuestión la idea de que el desarrollo del neoliberalismo, con sus grandes cuestiones problemáticas, ocurre de forma no intencional y con un carácter apolítico, no deseado, hasta aleatoriamente desde una aplicación autonomizada, propia de la era de la posmodernidad. Por el contrario, expresan que el contexto actual se construye en torno a la diversificación radical del capital económico y financiero que sienta las bases para la expansión de determinados aspectos que se solidifican formando un bloque que se presenta como un modo natural del devenir histórico; abarcando un gran número de categorías de percepción del mundo social para establecerse como "lo único posible," "un mundo en sí mismo," autogestado, dejando muy poco margen de actuación ante la imposición, sutil y engañosa, del consumo de mercancías.

Bajo el influjo de la ideología de libre mercado, se muestra la autonomía del capital con respecto al Estado; sin embargo entendemos que de esta manera se pretendería ocultar las modificaciones de las intervenciones estatales. Lo predominante sería entonces, aunque se condiga con los hechos acontecidos, una economía global que se apoya en la idea de una economía sin influencia del Estado. Esta coyuntura no se vincula únicamente, en palabras de Quiroga, "con la oposición entre producción social y apropiación privada, implica también la contradicción entre producción y consumo, capital productivo capital financiero, países centrales, países dependientes, mundialización y regionalización, entre otras" (2005, p. 21).

Se entiende que el análisis de estos fenómenos ayudará a comprender cómo estos movimientos geopolíticos a nivel global, que se están produciendo en estos tiempos, impactan en los países latinoamericanos de economías dependientes, dejando huellas que van a marcar la vida cotidiana de las comunidades y grupos que se pretenden analizar.

En suma, estos movimientos, si bien se presentan con un dinamismo propio, abarcan a los sujetos a la vez que los trasciende. En una entrevista nos decían:

"Hace veintiocho años que trabajo acá. (...) En ese entonces la mayoría era ferroviario y ahora no, muchísimo cambió, en el grupo de cooperadora la mayoría eran ferroviarios, la mayoría. (...) En esa época colaboraban mucho más. (...) Mi hermano puso todos los cerámicos que vos ves, sábado a la tarde y domingo venían a trabajar, ahora no viene nadie..." (Entrevista a una portera de la escuela y ex miembro del club de madres). 
Las organizaciones como instancias formadoras de lo grupal - sindicatos, escuelas, etc.- fueron el blanco del neoliberalismo que apuntó a la destrucción de lo colectivo como forma de la vida cotidiana. La consumación final produce, como observamos en la entrevista, un nuevo modo de relación entre el sujeto y las organizaciones sociales a las que pertenece. Junto a ello se va produciendo un movimiento de desvinculación y revinculación hacia otro espacio diferente al de la sociedad industrial del Estado de bienestar. (Beck, 1997). ¿Cuál es el sentido de pertenencia actual del sujeto en las organizaciones?

En cuanto a las características socio culturales del sujeto los rasgos más destacados, se refieren a un sujeto disponible para conectarse con todo, de vínculos fluctuante, permanentemente necesitado de mercancía para consumir, precario, polivalente, flexible a todos los movimientos (Dufour, 2007). Desde estos planteos nos preguntamos $¿$ Se trata de un sujeto liberado de los grandes relatos religiosos y/o políticos? O se pretendería instalar este discurso del sujeto del aquí y ahora para borrar la propia historia como una forma de desprendimiento -de aquellos relatos que estuvieron en el lugar de las certezas como enunciados de fundamento - para rearmar nuevas sujetaciones instalando nuevos relatos, nuevas certezas, como lo puede ser el mercado.

En Piera Aulagnier los enunciados del fundamento pertenecen al discurso del grupo social al que pertenece un sujeto.

Cualquiera que sean sus diferencias, estos enunciados comparten una misma exigencia: Su función de fundamento es una condición absoluta (...) Para que estos enunciados ejerzan tal función se requiere que puedan ser percibidos como palabras de certeza: de no ser así, serán dejados de lado y reemplazados (...) El discurso del conjunto le ofrece al sujeto una certeza acerca de su origen, para que sea retroactivamente proyectable sobre su pasado (...) El acceso a la historicidad es un factor esencial en el proceso identificatorio (1997, pp.160-164).

Sin un legado, se disuelven los vínculos de dependencias tradicionales, se pierde la transmisión del saber hacer, las creencias y las normas. Podríamos hablar de un sujeto desafiliado, que no está inscripto en ningún sistema social colectivo, sin descendencia, sin historia, producto de la individuación; ¿producto de nuevas certezas? En ese mismo desarrollo y posicionamiento como verdad absoluta, el sistema capitalista necesita que de la fluidez del capital, y para ello se ha instrumentado la posibilidad de "destruir" todo lo que pudiera desviar y demorar el movimiento libre del capital y limitar la libertad de mercado (Bauman, 2001) .

Las responsabilidades sociales ahora son transferidas a los sujetos que deben producir y constituir sus propias vidas, siendo los responsables únicos de ellas. En este sentido se establecen más exigencias para un sujeto libre, en el sentido de volátil, como desarmado aunque menos estable, cada vez más endeble y maleable con dificultades de afirmarse de manera íntegra; en 
este proceso -el que entendemos se encuentra en su etapa ascendente de afianzamiento- aunque nunca acabado ni lineal (Dufour, 2007).

Como consecuencia de ello, las leyes del sistema capitalista de producción - paralelamente productoras de las características mencionadas anteriormente de la sociedad actual- se filtran capilarmente convirtiéndose en lo que Bourdieu (1995) denominó habitus, estructura estructurante.

El habitus, es el producto de la interiorización de una multiplicidad de estructuras externas, El habitus es un operador de la racionalidad, pero de una racionalidad práctica, inmanente a un sistema histórico de relaciones sociales y, por ende, trascendente al individuo. Las estrategias por él gestionadas son sistemáticas pero también ad hoc, en la medida en que son desencadenadas por el encuentro con un campo particular. El habitus es creador, inventivo, pero dentro de los límites de sus estructuras (Bourdieu, 1995, p. 25).

Una vecina del barrio nos relataba: "Cuando yo vine de Italia en el 56' de a poco se fue haciendo (...) El barrio se fue poblando de a poco, venían de todos lados, extranjeros, paraguayos, del Chaco más que todos y de Santiago aunque eran pocos. (...)Venía uno y llama al otro se van llamando, por ejemplo una chica se casó y después llamó al hermano. Acá en el barrio había muchos metalúrgicos y ferroviarios que se fueron en la época de Menen. Mis sobrinos eran ferroviarios, cobraron la indemnización de veinte años, se fueron del barrio y no consiguieron más un trabajo seguro como el del ferrocarril."

En las dos entrevistas citadas hallamos el resultado producido por el despliegue de la vida cotidiana, con un sentimiento de ajenidad de las formas de vida, en el sentido de no llegar a percibir su articulación como fenómeno colectivo, para pasar a ser parte de lo naturalizado en el nivel de lo individual, como aquello que es frecuente de encontrar. Las percepciones del mundo social - las tipificaciones, los procedimientos interpretativos, las visiones valorativas y estimaciones estandarizadas-, por ser comunes a todos los sujetos estructurados en las divisiones sociales del orden social establecido, presentado anteriormente, se imponen con toda la apariencia del sentido común, son recibidas e incorporadas sin controversias, sin que puedan ser concebidas como tales quedando el sujeto en un estado de pasividad que conduce a la alienación como parte de la vida cotidiana. En relación a ello, la vida cotidiana es:

La forma de desenvolvimiento que adquiere día tras día nuestra historia individual, (...) nos muestra un mundo subjetivo (...) pero a la vez ese mundo es intersubjetivo, social y compartido, porque es un mundo que vivimos con otros (...). En la cotidianeidad las relaciones sociales se manifiestan y se ocultan. Se manifiestan en los hechos y se ocultan en la representación social de los hechos (Quiroga, 2003, p.12). 
Lo que caracteriza a la vida cotidiana es lo autoevidente, que es tomado como aquello de lo que no tiene sentido preguntarse; en su propio devenir se rodea de una familiaridad acrítica, que desde un orden natural encubre y distorsiona el carácter social de las manifestaciones concretas más arriba mencionadas (Ibídem).

\section{La identidad en juego o qué se juega en la identidad}

Para comenzar a definir la formación de las identidades desde una perspectiva sociológica, diremos que estamos refiriéndonos, en términos generales, a ponerle palabras a la pregunta por quién soy, que remite a una historia, a todas aquellas prácticas sociales que forman sujetos sociales. La construcción de la identidad es un trabajo que realiza el yo- como instancia psíquica- del sujeto que, en un trabajo activo, va tomando aspectos de la cultura y de lo histórico-social dando una respuesta, nunca acabada, a la pregunta anteriormente presentada: quién soy; a la que se le agrega, por considerarse que no se encuentran separadas, qué puedo hacer. La identidad en estos términos, es potenciada en el espacio público retroalimentándose constantemente.

La subjetividad - al igual que la identidad - se relaciona con los condicionantes de la existencia, está moldeada por lo socio - cultural y determinada por el momento histórico en el que transcurre; aunque la subjetividad es la cualidad en la que se plasma el psiquismo, el modo histórico de producción arroja determinadas singularidades psíquicas. De ahí que entendamos la constitución del sujeto y su subjetividad condicionada por lo sociocultural, lo histórico y libidinal (Grande, 2004).

En palabras de Ana Quiroga la identidad es "interrogarse acerca de quienes somos, que significamos para los otros, preguntarnos por nuestras relaciones, por nuestras identificaciones. Y pensar la identidad nos plantea reflexionar sobre nuestra historicidad subjetiva, familiar, social, preguntarse acerca de nuestros referentes, nuestras pertenencias, nuestros ideales" (1996, p. 21).

Existe una gran variedad de definiciones acerca de la concepción de la identidad. En este apartado hemos focalizado en dos grandes grupos de corrientes teóricas que, entendemos, representan propuestas diversas aunque se muestren aparentemente, con planteamientos similares. En estos dos grupos el concepto de identidad queda separado también de aquello que hace referencia a cuestiones del orden sobrenatural o metafísico, como algo que hay que descubrir en el interior espiritual de cada individuo así como de determinaciones naturales con las que el individuo nace. Se estaría planteando que la cultura - como proceso civilizatorio - muestra su producción plasmándose en la identidad de cada sujeto. En el primer grupo, la identidad aparece conformada a través de la acumulación histórica de elementos formados por ideas, sentimientos, prácticas simbólicas, costumbres, creencias y representaciones, produciéndose un sedimento que da como resultado la identidad de un sujeto. 
Allí, en una interacción mutua entre los sujetos, diferentes procesos del devenir social actuarían como "modificadores" de la identidad. La identidad no existiría como entidad estática y abstracta sino como producto de una construcción constante, en un proceso de cimentación y deconstrucción continua. Como las capas de una cebolla, envuelven al yo dándole forma de identidad.

En cuanto a lo grupal, pone el acento más en el poder de persuasión de los grupos que, como una "balanza," definen las identidades grupales, el nosotros que les da un sentimiento de mismidad, en oposición a los otros. Aparece el concepto de identidad como una construcción simbólica, desde un ordenamiento clasificatorio. Cada grupo crea su propia denominación desde la que encuentra su definición y simultáneamente los opone a otras agrupaciones. Los grupos se permiten redefinirse frecuentemente por lo que los límites entre unos y otros son endebles y fácilmente modificables. Foucault, (1997) y Deleuze, $(1968,1987)$ son los autores centrales de esta corriente de pensamiento; quienes conciben que la identidad procede de la diferencia, que es previa a esta. Comprenden a la diferencia como la afirmación de una multiplicidad por fuera de la primacía de lo idéntico: El otro no es "otro como yo" o "como uno" es absolutamente "otro" y de un modo inconmensurable.

En un segundo grupo encontramos que, si bien hay acuerdo en plantear el proceso de sedimentación como forma constitutiva de la configuración de la identidad, a la vez que es esa misma acumulación de elementos es la que permite la diferenciación de unos con otros y la posibilidad de armar una grupalidad; estos procesos no se presentan aisladamente y sin contrariedades. A diferencia de estas líneas teóricas, que abordan el concepto de identidad quitándole la faz conflictiva al proceso de sedimentación, el enfoque de análisis que presenta este grupo -y que proponemos para el sucesivo análisis- plantea que el entramado social no puede ser abordado con independencia del interrogación histórica en el cual se origina, a la vez que lo individual es abordado en ese encuentro de la historia social como producto y consecuencia de la estructura social.

La identidad es el resultado de un proceso de constitución continua, durante el cual diversos elementos contradictorios no solo se unen sino que se mantienen en tensión y disputa. En este proceso hay cambios y continuidad. Y se va conformando tanto en cada individuo, como en lo colectivo una totalidad de elementos que permiten a la comunidad y a cada uno de sus miembros, identificarse a la vez que diferenciarse. Estamos refiriéndonos a la continuidad histórica de una comunidad relativamente estable, que a lo largo del tiempo gesta un complejo de culturas, de lengua, de psicología, que adquiere conciencia de sí y de los otros como diferente y semejante (Racedo, 2004, p. 21).

En el revés de la trama social se asienta la formación de las identidades de una época, desterrándola así de cuestiones que hacen a un orden natural, para ubicarla en un proceso histórico de lo social. 
Una entrevistada narraba: "En el 55' mis padres vinieron a vivir al barrio, mi papá era de Córdoba y mi mamá de Entre Ríos. (...) Mi papá es socio fundador de la vecinal, se reunieron entre cinco y sacaron un crédito en el Banquito Ferroviario para poder hacer la vecinal. Ellos siempre con la idea del saloncito y el asado para reunirse (...) La gente se cree que esto se hizo de un día para el otro, (...) las pocas personas que estamos acá tenemos puesta la camiseta de la vecinal si no, no iría ni para atrás ni para adelante. -: Es como que recibiste un legado....: ¡Si!... ¡y que legado! ... ¡Y como pesa!" (Entrevista a un miembro de la comisión de la vecinal del barrio).

En estas expresiones vemos como la configuración de la identidad, se plasma como una síntesis. Síntesis que, por un lado muestra, las características del sujeto, aquello que lo singulariza, y una configuración identitaria, envuelta y estructurada, por la historia social del barrio, como un producto de esa relación/tensión que establece el sujeto con el contexto histórico en el cual se desarrolla; en otras palabras, podemos distinguir y relacionar a la identidad con lo estrictamente histórico y social de un sujeto. Junto a ello, consideramos que la configuración de la identidad encierra no solo una trama de problemáticas intrínsecas, propias del sitio que se ocupa en una época y del recorrido singular, sino que existen determinaciones por fuera del sujeto, hasta muy sutiles -extrínsecas, condicionándolas estructuralmente- sin que, muchas veces, este fenómeno pueda ser considerado por los propios sujetos de forma consciente como un acontecer restrictivo propio de las identidades. En relación a ello, pensamos en el "peso del legado," al que se hace referencia la entrevistada, y el énfasis en resaltarlo; como si algo del orden del legado de una herencia cultural y del compromiso por haberla recibido se estuviera poniendo en juego; a ello Piera Aulagnier lo llamó contrato narcisista:

El contrato narcisista se instaura como un pacto de intercambio: el grupo garantiza la transferencia sobre el nuevo miembro del reconocimiento (...) el nuevo miembro se compromete (...) a repetir el mismo fragmento de discurso, (...) que repita los enunciados, garanizando así, la permanencia del grupo. El niño demandará que se le ofrezca un modelo ideal (...) que le permita conservar la ilusión de una persistencia atemporal sobre el conjunto y, (...) que según supone sus sucesores retomarán y preservarán (1997, pp.163-164).

Por otro lado, es una síntesis integrada a un entramado de vínculos referenciales del barrio. Elias (1987) define a los vínculos referenciales, en relación a una cualidad histórica y social de su grupo de pertenencia como concluyente en la configuración identitaria. Utiliza el concepto de configuración como un entramado que es propio de los seres humanos y que solo se da entre ellos como una especie de ligazón de sus interdependencias, formándose los vínculos de unos con otros, teniendo siempre en cuenta que estas relaciones entre los individuos son siempre sociales y se desarrollan a través del tiempo por largos períodos. De esta manera se encuentra al individuo y a la sociedad como entidades que se retroalimentan constantemente. 
Partiendo de este señalamiento, encontramos a la identidad integrada por la contradicción existente entre lo individual y lo colectivo; como dos niveles de determinación que se cruzan y tensionan entre sí. Desde lo individual, como ya dijimos, entendido como lo histórico singular, se hace referencia, a los procesos de constitución psíquica de un sujeto particular apuntando tanto a los procesos identificatorios como a la construcción de ideales; y desde lo social colectivo nos referimos al escenario concreto del desarrollo de la existencia con su correlato en la producción y reproducción de formatos sociales. Al respecto Norbert Elias expresa:

Se usan habitualmente los conceptos "Individuo" y "sociedad", como si se tratara de dos sustancias pasivas distintas. Al emplear así estas palabras, se suscita fácilmente la impresión de que aquello que denotan, no solo son objetos distintos, sino que existen absolutamente separados; en realidad son procesos que, sin duda alguna, pueden distinguirse, pero no separarse (1996, p.33).

Es interesante también, tomar la propuesta de Racedo (2004) en relación al vínculo entre el individuo y sociedad, cuando habla de la conformación de una unidad de opuestos en el interior de la identidad. Allí interviene, un juego de lucha dialéctica entre, podríamos decir un polo que denomina la identidad asignada y otro polo la identidad emancipada. La primera especificada por los valores y discursos de la clase dominante; en este sentido, señalada desde el deber ser, el ser nacional, y la segunda que se construyó y se construye desde la concientización del lugar que se ocupa en la estructura del sistema de dominación sin aceptar dicha posición. Esta autora, a diferencia de los planteos de los autores del primer grupo, habla, entonces de una dialéctica en el núcleo de la configuración de la identidad en donde se registran dos aspectos: uno donde se juegan elementos de los sectores dominantes y el otro los elementos de resistencia. De la lucha y tensión resulta la síntesis que denominamos identidad entre lo asignado y lo emancipatorio, desde ahí es que significamos y resignificamos el mundo construimos nuestro esquema referencial de sentidos del mundo que le da razón de ser a la propia identidad.

Interesa destacar, en relación a estos planteos, cómo la noción de normalidad/anormalidad va participando, desde lo histórico social como una característica central de la configuración de la identidad y en la edificación de los cimientos culturales; que al nombrar nuevas formas de "lo adecuado" y lo más "óptimo", del mismo modo, estructura los principios de lo bueno/malo de la salud/enfermedad en los diferentes sectores sociales. Es interesante examinar cuál es el polo que predomina, por cuánto tiempo se instala esta supremacía de uno sobre el otro, cómo se generan las condiciones para el movimiento de desestructuración y reestructuración en tanto que el predominio de un polo sobre el otro no es uniforme entendemos pueden producirse un desacomodamiento y reacomodamiento que reestructure la configuración de la identidad. 


\section{La identidad de los sectores populares en las prácticas educativas}

Nos preguntamos como la escuela puede sostener y ofrecer un proyecto, cuando las identidades se están configurando actualmente, bajo el predominio de la incertidumbre, y cómo podrá sostenerse un proyecto integral, a futuro, en este presente que reúne la fragmentación y la fluidez en los vínculos, como elementos instalados en la vida cotidiana. Aulagnier denomina al proyecto identificatorio como "el soporte de las identificaciones que (...) el sujeto debe encontrar en el discurso de su grupo de pertenencia, referencias que le permitan proyectarse hacia un futuro" (1997, p.159).

¿Estaremos en presencia de un proceso de afianzamiento de la globalización de lo cultural con implementación en las organizaciones escolares que busca homogeneizar para igualar ciertos cánones culturales universalmente aceptados para las nuevas generaciones? ¿El proyecto educativo de la globalización, que borra las diferencias con las que parten los niños de sectores populares, podría estar generando discursos falaces en torno al acceso y la permanencia en el sistema educativo formal?

Además, si el escenario económico político que presentamos se muestra como una implacable transformación del sustrato cultural, cómo podrán las comunidades y grupos sostener sus propias identidades históricamente definidas. En las diferentes realidades sociales se viene experimentando con mayor o menor intensidad una pérdida del sentido de pertenencia provocando profundos conflictos.

Dentro del sistema escolar, Bourdieu y Wacquant (1995), expresan que existirían acciones pedagógicas destinadas a reproducir cánones culturales que corresponden a los intereses de las clases dominantes, de ahí que el trabajo pedagógico escolar tendría una productividad diferencial de acuerdo a la clase social de origen de los individuos. Para los que provienen de los sectores dominantes, la educación escolar será una re- educación, mientras que para quienes pertenecen a los sectores dominados, tendrá el sentido de la marginación.

Entendemos que no solo existen distinciones que pertenecen a cada grupo de referencia, sino que desde estos contrastes, formas de vida, de representación del mundo, surge la posibilidad de configurar la identidad social del grupo de pertenencia/referencia.

En la escuela, junto al trabajo docente de desarrollo de las potencialidades del pensamiento, es interesante no desconocer, ni dejar de lado lo que se relaciona con la identidad del grupo social en el que está inserta como institución educativa; tomando los enunciados de fundamentos propios de quien se encuentra en el lugar de alumno, ello no significa interpretar la singularidad sino, por el contrario, encarna la posibilidad de potenciar las identificaciones. Más que homogeneizar adaptativamente, intentamos el rescate de las identi- 
dades del legado cultural que corresponden al discurso del conjunto social de pertenencia; se trata de afirmar las diferencias en la integración.

Si la escuela parte de la transmisión sólo de la cultura hegemónica aunque amplíe los niveles estandarizados de lo que denominamos cánones culturales poco podrá hacer para el despliegue y afirmación de la identidad de los niños que quedan fuera de la cultura hegemónica, en cambio una escuela inclusiva que parta, para su proyecto educativo teniendo en cuenta el espacio socio- histórico de pertenencia, de la realidad concreta en donde está inserta para construir su proyecto curricular, realiza un viraje hacia las propuesta que contemplan el entorno como factor determinante de las prácticas. Quizá partiendo desde ahí hacia la reconstrucción de significados que hacen a lo escolar, se puedan rescatar proyectos identificatorios del conjunto social, resignificar el lugar de la transmisión solo de cánones culturales de una identidad asignada y externa a los sectores populares; y finalmente, que tiendan a la edificación de una identidad emancipada (Rosbaco, 2000; Schlemenson, 1996).

De ahí se inicia y avanza la escuela aunque no queda estancada allí, debe transmitir la ciencia, la tecnología, como herramientas que permitan a los sujetos que la integran poner en juego sus deseos y necesidades, para que en este proceso puedan dotar de sentidos también emancipadores para la escuela (Freire, 1993).

\section{Reflexiones finales}

Para finalizar, quisiéramos hacer mención, a modo de cierre o de síntesis parcial, a ser retomada en próximos escritos, de algunos puntos esbozados que nos parece interesante destacar. El proceso del presente trabajo pudo ser posible porque hay un contexto de desarrollo científico de las Ciencias Sociales y Humanas que permite pensar epistemológicamente ciertas problemáticas, ampliando la visión de los campos disciplinares.

El concepto de identidad es un elemento fundamental para rescatar en las prácticas escolares de los sectores populares, desde el trabajo con los enunciados de fundamento del discurso social del grupo de pertenencia, en el que están insertas.

Buscamos poner en duda ciertas imposibilidades que se marcan de la escuela, instalándola en el lugar solo de reproductora de desigualdades sociales; para pensarla como transmisora de conocimientos y potenciadora de la identidad.

Entendemos que la síntesis superadora del presente es aquella que siempre está por alcanzarse, es la que se encuentra en construcción permanente, la que muta de acuerdo a aquello que nace, que se crea y se refunda constantemente. El pensamiento en un persistente devenir es el que habilita nuevos parámetros atento a los desafíos diarios de la educación actual, trasuntando con ello en la movilización de aquellas estructuras otrora inalterables. 


\section{Referencias Bibliográficas}

- $\quad$ Aulagnier, P. (1997) La violencia de la interpretación. Del pictograma al enunciado. Argentina, Amorrortu.

- Bauman, Z. (1999) La globalización. Consecuencias Humanas. Argentina, Fondo de Cultura Económica.

mica.

(2001) En busca de la política. Buenos Aires, Fondo de Cultura Econó-

- $\quad$ Beck, U.; Giddens, A.; Lash, S. (1997) Modernización reflexiva: política, tradición y estética en el orden social moderno. Madrid, Alianza.

- Beck, U. (1998) La sociedad del riesgo. Hacia una nueva modernidad. Buenos Aires, Paidós.

- $\quad$ Berger, P.; Luckman, T. (1997) Modernidad, pluralismo y crisis de sentido. La orientación del hombre moderno. Buenos Aires, Paidós.

- $\quad$ Bixio, B. Heredia, L. (2000) Algunos lugares de articulación disciplinaria: La vulnerabilidad de las fronteras en Revista Publicación del CIFFyH Interdisciplina $N^{\circ} 1$, Año 1, marzo pp. 83-94. ISSN 1515-1859. Córdoba, Centro de Investigaciones Facultad de Filosofía y Humanidades, Universidad Nacional de Córdoba.

- Bourdieu, P. Wacquant, L.J.D. (1995) Respuestas por una Antropología reflexiva. México, Grijalbo.

- $\quad$ Bourdieu, P. (1997) Capital cultural, escuela y espacio social. México, Siglo Veintiuno.

- Deleuze, G. (1968) Difference et repetition. París. Presses Universitaries de France. (1987) Foucault. Argentina, Paidos.

- Dufour, D. (2007) El arte de reducir cabezas. Sobre la servidumbre del hombre liberado en la era del capitalismo. Buenos Aires, Paidós.

- $\quad$ Elias, N (1987) El Proceso de la civilización. México, Fondo de Cultura Económica.

- - (1996) La sociedad cortesana. México, Fondo de Cultura Económica.

- $\quad$ Forlini, N. (2006) El pensamiento propio en niños marginales. Propuesta para potenciarlo. Tesina de Licenciatura no publicada, Facultad de Humanidades y Artes, Universidad Nacional de Rosario,

- $\quad$ Foucault, M. (1997) Las palabras y las cosas. Madrid, Siglo XXI.

- Freire, P. (1993) Pedagogía de la esperanza. Un reencuentro con la pedagogía del oprimido. Buenos Aires, Siglo XXI.

- Grande, A. (2004) Psicoanálisis implicado 3. Del diván al piquete. Argentina, Topía.

- Giddens, A. (2002) Modernidade e identidade. Rio de Janeiro, Jorge Zahar Ed.

- Heinich, N. (1999) Norbert Elias Historia y cultura en occidente. Bs. As., Nueva Visión.

- Heler, M. (2004) Ciencia incierta. La producción social del conocimiento. Bs. As., Biblos.

- Mitjavila, M. (2006) El riesgo como instrumento de individualización social, en Burkún, M. y Krmpotic, C. (comp.) El conflicto social y político. Grados de libertad y sumisión en el escenario local y global, pp. 49-62. Buenos Aires, Prometeo.

- Quiroga, A. (1996) “Identidad y realidad social”, en Revista Temas de Psicología Social. $\mathrm{N}^{\circ}$ 15. Año 17, pp 19-32 ISSN 0326-453 .Bs. As, Cinco.

(1998) Enfoques y Perspectivas en Psicología Social. Desarrollos a partir del pensamiento de Enrique Pichon - Riviere. Bs. As, Cinco.

(2005) Crisis, Procesos sociales y grupo. Desarrollos en psicología social a partir del pensamiento de Enrique Pichon - Riviere. Bs. As, Cinco.

- Racedo, J.[et al] (2004) Patrimonio cultural e identidad. Culturas populares, memoria social y educación. Bs. As, Cinco. 
- Requejo, M. I. (2004) Lingüística social y autorías de la palabra y el pensamiento. Temas de debate en Psicología Social y Educación. Buenos Aires, Cinco.

- Rosbaco, I. (2000) El desnutrido escolar. Dificultades de aprendizaje en los niños de contextos de pobreza urbana. Rosario, Homo Sapiens.

- - (2003) Constitución del pensamiento relativamente autónomo: incidencia de la estructura narrativa. Tesis Doctoral no publicada, Facultad de Psicología, Universidad Nacional de Rosario.

- $\quad$ Schlemenson, S. (1996) El aprendizaje, un encuentro de sentidos. Buenos Aires, Kapeluz. 